\title{
Las ciencias de la naturaleza a través de los cuentos eneducación infantil
}

\section{Nature sciences through storytelling in early childhood education}

\author{
DOI: $10.46932 /$ sfjdv2n5-023
}

Received in: Jun 1st, 2021

Accepted in: Sep 30th, 2021

\author{
Estela María Celdrán Fernádez \\ Grado en Educación Infantil \\ Olga Quirante Martínez \\ Grado en Educación Infantil
}

\begin{abstract}
RESUMEN
El objetivo general de este artículo consiste en analizar la puesta en práctica de una secuencia de enseñanza sobre ciencias de la naturaleza en la que se utiliza el cuento como recurso didáctico principal. Dicha propuesta tiene como objetivo trabajar el modelo de ser vivo a través de los pollitos y realizar una analogía con el ser humano. Esta propuesta está diseñada para un aula del segundo ciclo de educación infantil (3 años), perteneciente a un colegio público de la Comunidad Autónoma de la Región de Murcia, con 8 niñas y 16 niños, en total 24. La metodología que enmarca esta secuencia didácticaes el trabajo por proyectos, aprovechando que en el aula se estaba viendo un proyecto sobre los pollitos y, para ello, se hace uso del cuento de "El pollo Pepe" cuyo autor es Nick Denchfield. En el apartado de: reflexión sobre la puesta en práctica, se recogen unaserie de resultados favorables en cuanto a esta propuesta, lo que significa que es una secuencia adecuada y viable para estas edades, en la que se consigue un acercamiento a las ciencias de la naturaleza a través de la literatura infantil.
\end{abstract}

Palabras clave: ciencias de la naturaleza, secuencia de aprendizaje, educación infantil,cuento, seres vivos

\begin{abstract}
The general objective of this article is to analyze the implementation of a teaching sequence on natural sciences in which the story is used as the main didactic resource. The objective of this proposal is to work on the model of a living being through the chicks and to make an analogy with the human being. This proposal is designed for a classroom of the second cycle of early childhood education (3 years old), belonging to a public school of the Autonomous Community of the Region of Murcia, with 8 girls and 16 boys, in total 24 . The methodology that frames this didactic sequence is the work by projects, taking advantage of the fact that in the classroom a project about chicks was being seen and, for this, the story of "El pollo Pepe" whose author is Nick Denchfield is used. In the section on: reflection on the implementation, a series of favorable results are collected regarding this proposal, which means that it is an appropriate and feasible sequence for these ages, in which an approach to the sciences of nature is achieved through children's literature.
\end{abstract}

Keywords: nature sciences, learning sequence, children's education, story, living beings. 


\section{INTRODUCCIÓN}

Este artículo versa sobre cómo enseñar las Ciencias de la Naturaleza en EducaciónInfantil a través de la literatura infantil: los cuentos.

En las aulas de este rango de edad comprendido entre los 3 y 5 años, no siempre se trabaja este campo de estudio de la manera adecuada, la cual debería ser eminentementepráctica en la medida de lo posible en tanto que las circunstancias y características del aula así lo permitan. Trabajar los contenidos de manera teórica puede resultar poco alentador, lo que conlleva que el alumnado deje de prestar atención y no muestre interés.En cambio, con actividades estimulantes podremos lograr que todos participen y se muestren expectantes ante ese tipo de ejercicios, logrando así, por tanto, una mejora en ladinámica del aula.

En ocasiones, esta metodología tradicional se da en aquellos colegios que se encuentran ubicados en zonas urbanas, en las que no disponen de la naturaleza propiamente dicha. Podemos encontrar propuestas en las que únicamente se rellenan fichas y, de vez en cuando, se utilizan técnicas plásticas para ello. Pero esto, no deja de ser lo mismo de siempre, contenidos teóricos con poco margen de actuación práctica. Sinembargo, la importancia reside en encontrar una armonía y un balance entre lo teórico y lo práctico, en el que los alumnos y alumnas van a aprender y disfrutar de las ciencias.

No es necesario llevar al alumnado a una granja para que conozca el modelo de ser vivo. Basta, simplemente, con salir al patio del colegio y observar, por ejemplo, las hormigas que van por el suelo, los saltamontes o las plantas del huerto, en caso de que haya.

Es por todos sabida la curiosidad innata que sienten los niños y niñas sobre la naturaleza y todo lo que la rodea. Por eso, debemos aprovechar este interés para promoversu acercamiento y sacar el máximo partido.

Dentro de este campo tan amplio, se pueden encontrar diversos temas sobre los que hablar y debatir con los alumnos y alumnas, así como una serie de métodos de aprendizaje que podemos poner en práctica según los intereses y necesidades. Algunos ejemplos de ello son:

- Centros de interés.

- Trabajo por proyectos.

- Aprendizaje basado en problemas.

- Aprendizaje basado en retos.

- Aprendizaje servicio.

En este caso, esta propuesta didáctica se puso en práctica en un aula de 3 años donde se estaba trabajando por proyectos, en concreto, uno sobre los pollitos. Se entiendepor trabajo por proyectos, según 
Muñoz y Díaz (2009) como "una opción metodológica basada en la investigación - acción, cuyo objetivo es organizar los contenidos curricularesbajo un enfoque globalizador y significativo, relacionando los conocimientos escolares con los de la vida cotidiana" (p. 106). De esta manera, las actividades propuestas se encuentran ubicadas bajo esta metodología, con el fin de poder integrarlas sin alterar el ritmo de trabajo que estaban siguiendo.

Un cuento que encaja en dicho proyecto y con el que se puede lograr que los niñosy niñas aprendan y afiancen más conceptos sobre los pollitos es "El pollo Pepe", cuyo autor es Nick Denchfield, su ilustrador Ant Parker y ha sido producido por la editorial SM.

Este cuento relata, de manera visual y divertida, cómo es el cuerpo de un pollito, lo que come y una comparación de tamaño con su mamá: la gallina. Además, pese a quees un cuento simple, guarda unas sorpresas en cada una de sus páginas a través de desplegables, por lo que resulta atractivo y llamativo para los alumnos y alumnas que lo ven. Tiene muchas posibilidades en cuanto a los contenidos que podemos trabajar a travésdel mismo, que especificaremos más adelante. Por tanto, la secuencia de aprendizaje utiliza como hilo conductor al pollo Pepe, como protagonista de un cuento para enseñar ciertos aspectos de las Ciencias de la Naturaleza.

El pollito es un animal que los niños y niñas relacionan con el medio, por lo que no es un concepto aislado que no puedan comprender o les resulte difícil de identificar. De hecho, justo el primer día que se comenzó la secuencia, un alumno del aula de 5 años llevó dos pollitos para enseñárselo a sus compañeros y compañeras, por lo que los niñosy niñas de 3 años también pudieron observar de manera breve, pero de primera mano, cómo es un pollito: su tamaño, su color o su interacción con el medio.

Este aspecto se encuentra enmarcado en el Decreto 254/2008 de 1 de agosto por el que se establece el currículo del segundo ciclo de la Educación Infantil en la Comunidad Autónoma de la Región de Murcia, dentro del área de Conocimiento de Entorno, referido al Bloque 2. Acercamiento a la naturaleza.

\section{MARCO TEÓRICO}

Puesto que el recurso sobre el que trabaja en la secuencia de aprendizaje es el cuento, debemos de conocer una serie de cuestiones al respecto: ¿qué significa la palabracuento?, ¿qué estructura presenta?, ¿qué tipos de cuentos podemos encontrar?, ¿qué beneficios aporta en estas edades?, ¿por qué enseñar ciencias?, ¿de qué manera influye en el aprendizaje de las ciencias de la naturaleza?

En cuanto a su definición, podemos encontrar las aportaciones de diversos autoresen la siguiente tabla: 
Definición de cuento según diversos autores.

\begin{tabular}{|c|c|c|}
\hline Autor & Fecha & Definición \\
\hline Cabrera & 2001 & $\begin{array}{l}\text { El cuento es tan antiguo como el hombre. Tal vez incluso másantiguo, pues } \\
\text { bien pudo haber primates que contaran cuentos todos hechos de gruñidos, } \\
\text { que es el origen del lenguaje humano: (...). Así nació la onomatopeya y } \\
\text { con ella, luego, la epopeya. Pero antes que ella, cantada o escrita, hubo } \\
\text { cuentos todos hechos de prosa: un cuento en verso no es un cuento sinootra } \\
\text { cosa: un poema, una oda, una narración con metro y tal } \\
\text { con rima: una ocasión cantada no contada, una canción. }\end{array}$ \\
\hline Imbert & 1979 & $\begin{array}{l}\text { El cuento vendría a ser una narración breve en prosa que, pormucho que } \\
\text { se apoye en un suceder real, revela siempre la imaginación de un narrador } \\
\text { individual. La acción - cuyos agentes son hombres, animales } \\
\text { humanizados o cosas animadas- consta de una serie de acontecimientos } \\
\text { entretejidos en una trama donde las tensiones y distensiones, graduadas } \\
\text { para mantener en suspenso el ánimo del lector, terminan por resolverse } \\
\text { en un desenlace estéticamente } \\
\text { sfactorio (p. 35). }\end{array}$ \\
\hline
\end{tabular}

Real

Academia

Española

(RAE)
Podemos encontrar tres acepciones:

1. Narración breve de ficción.

2. Relato, generalmente indiscreto, de un suceso.

3. Relación, de palabra o por escrito, de un suceso falsoo de pura invención.

Fuente: elaboración propia.

En cuanto a la estructura del cuento, podemos diferenciar tres partes claras: la primera, en la que se presenta a los personajes en un lugar concreto, la segunda parte hacereferencia al desarrollo como tal del cuento, en el que sucede algo que provoca que los personajes se encuentren ante un problema al que deben buscar una solución y, en tercerlugar, el desenlace del mismo, que generalmente suele ser un final feliz, en el que los personajes encuentran la manera de solucionar el problema (Espinet Blanch, 1995).

Según Toledo Morales (2005), en lo que respecta a los tipos de cuento, podemos hacer una clara distinción entre: cuentos orales o populares y cuentos literarios. Por un lado, los cuentos populares se remontan a muchos siglos atrás y se conforman, en definitiva, de los hábitos, dichos o rituales que sean propios de la época. Son de tradiciónoral y no hay una única versión ya que dependerá, como se ha mencionado, de la época en la que se cuenten. Por otro lado, podemos hablar de los cuentos literarios, los cuales son plasmados en papel, pueden estar basados en cuentos de tradición oral y tienen un texto definido.

De acuerdo con Fernández Serón (2010), el cuento presenta diversos beneficios que debemos tener en cuenta para su elección, en función de lo que pretendamos conseguir con él. No solo logramos que el niño o la niña lo disfrute, sino que conoce diversos personajes que le aportan enseñanzas. En adición, aunque los infantes no sabenleer en estas edades, se produce un primer acercamiento a la 
lectoescritura, ya que van comprobando las diferencias que puede haber entre los números y las letras, así como comenzar a distinguir mayúsculas y minúsculas.

Además, a través de los cuentos podemos enseñar multitud de contenidos. Las vivencias que aparecen en el relato pueden ser cercanas al alumnado y enseñar valores tales como la empatía, la generosidad, la amistad, el esfuerzo, la sinceridad, la tolerancia o la paciencia, así como conductas que no deben reproducir en su día a día, como decir mentiras o tener comportamientos inadecuados con los demás (Martínez Urbano, 2011).

No podemos olvidar, no obstante, que a través de los cuentos también podemos educar en educación emocional, muy importante y necesario en estas edades tempranas. Según López Cassá (2016), "las emociones están presentes en nuestras vidas desde que nacemos y juegan un papel relevante en la construcción de nuestra personalidad e interacción social” (p. 154). Por tanto, los niños y niñas pueden verse reflejados en los personajes de los relatos en cuanto a la emoción que estén sintiendo: alegría, tristeza, enfado, miedo..., por lo que es una manera ideal de que ellos sean conscientes de que todos y cada uno de los sentimientos que perciben son necesarios para la vida y no tenemos por qué esconderlos.

Ahora bien, teniendo en cuenta el objetivo de esta secuencia, se formula la siguiente cuestión: ¿por qué enseñar ciencias?

Según Veglia (2007) "la ciencia es una manera de mirar el mundo y de reflexionarsobre él” (p. 19). Su enseñanza debe ir más allá de la transmisión de conocimientos, paraconseguir que los infantes disfruten de su entorno. Se fomenta, además, la reflexión del mismo, una visión crítica que otorga la capacidad de resolver situaciones o conflictos. Por tanto, en palabras de Veglia (2007): “a través de las ciencias se puede colaborar a mejorar la calidad de vida de las personas, a través de pautas que los ayuden a tomar decisiones responsables" (p. 19), en relación a una serie de aspectos tales como la salud propia o el cuidado del medioambiente.

Sin embargo, no se pueden transmitir los conocimientos puramente científicos a los alumnos y alumnas, pues poseen unas cualidades que resultan complejas a los ojos delos discentes de estas edades. El paso del modelo científico a ciencia escolar se realiza a través de la transposición didáctica, entendida según Chevallard (1991), citado por Gutiérrez Abbad, A. (s.f.). como: "la transformación del saber científico en un saber susceptible de ser enseñado".

De esta manera, una vez que los contenidos están adaptados, es momento de enseñarlos a nuestro alumnado. En este caso, a través de los cuentos.

Por tanto, ¿de qué manera influyen los cuentos en el aprendizaje de las Ciencias de la Naturaleza? Enseñar ciencias naturales, de acuerdo con Pérez Molina, Pérez y Sánchez (2013)“no significa 
únicamente enseñar flora, fauna, o medios de transporte o cualquier otro contenido de manera exclusivamente teórica, sino que esta actividad puede variar de muchas maneras" (p. 04). Es decir, no existe un listado de cosas que debemos enseñar a los alumnos y alumnas, sino que es un compendio de conocimientos entrelazados entre sí, que debemos abordar de diferentes maneras para que resulte motivador.

El cuento favorece la enseñanza de dichos contenidos porque fomenta la interacción entre el docente y el alumnado. Para que dichas interacciones puedan darse, debemos escoger un cuento sencillo, especialmente en el segundo ciclo de Educación Infantil. Es el docente quien debe tener la competencia de escoger el cuento en base a lascaracterísticas de su alumnado y los contenidos que se pretenden mostrar con él (Pérez Molina, et al., 2013)

Asimismo, Pérez Molina, et al. (2013) afirman que:

Enseñar conocimiento del medio no implica solamente explicarles a los niños aspectos de la vida cotidiana mencionados en el currículum, sino que esto implica que estos contenidos tengan que ser tratados a partir de una relacióncon otras materias, como por ejemplo la lengua y la literatura. Los cuentos, además de mejorar las capacidades lingüísticas, también facilitan e incrementan la adquisición de contenidos (tanto de ciencias naturales como de cualquierotra materia) (p. 05).

Por consiguiente, podemos asegurar que el cuento es un recurso educativo con grandes posibilidades en cuanto a la enseñanza de las ciencias en estas edades, por la multitud de beneficios que presenta y por lo novedoso que resulta, alejándonos, por tanto,del método tradicional con el que se han enseñado las ciencias durante décadas.

El objetivo que se pretende conseguir, por tanto, es aplicar la secuencia de aprendizaje en el aula para trabajar el concepto de ser vivo a través de los pollitos y el cuento, además de elaborar una analogía entre los distintos aspectos trabajados con el serhumano, que se corresponden con los objetivos propuestos en la secuencia de aprendizaje, los cuales se especifican en siguientes apartados.

\section{ESTUDIO REALIZADO}

\subsection{CONTEXTO Y PARTICIPANTES:}

El centro en el cual se ha llevado a cabo la propuesta que se describe en este trabajose encuentra ubicado en la Región de Murcia, a pocos kilómetros del centro de la ciudad.Las familias del alumnado se dedican principalmente al sector servicios, por lo que podemos afirmar que el nivel socioeconómico de las mismas es medio-alto.

El aula se compone de 24 alumnos y alumnas: 8 niñas y 16 niños. Las mesas se encuentran dispuestas en grupos de trabajo, en total cinco, referidos cada uno de ellos a un color distinto, por tanto, 
el alumnado trabaja de esta manera. La dinámica de la clase se guía a través del trabajo por proyectos, como comentamos en apartados anteriores. Además, el aula presenta cinco rincones a los que el alumnado acudirá una vez realizadala actividad de trabajo propuesta.

Estos rincones son:

- $\quad$ Rincón de la biblioteca.

- $\quad$ Rincón de los puzles.

- $\quad$ Rincón de la casita.

- $\quad$ Rincón de la plástica.

- $\quad$ Rincón de las construcciones.

En ellos, podemos encontrar todos los materiales relacionados con cada uno. Un ejemplo de ello serían los bloques lógicos o las regletas de Cuisenaire. En cuanto a los recursos humanos, podemos encontrar que al aula acuden diferentes profesionales: maestras de apoyo, maestra de segunda lengua extranjera: inglés y maestra de religión.

\subsection{PLANIFICACIÓN Y DISEÑO DE LA ACCIÓN DOCENTE:}

Siguiendo las orientaciones metodológicas que podemos encontrar en el Decreto254/2008 de 1 de agosto por el que se establece el currículo del segundo ciclo de la Educación Infantil en la Comunidad Autónoma de la Región de Murcia, podemos decir que la metodología que se emplea para llevar a cabo esta propuesta didáctica se basa enlos siguientes aspectos:

Por un lado, debemos tener en cuenta las necesidades individuales del alumnado,con el fin de organizar nuestra docencia hacia la flexibilidad, la atención individualizaday las necesidades específicas que puedan surgir en determinados momentos.

Para ello, resulta necesario conocer las características de los niños y niñas, para lograr aprendizajes significativos, los cuales se dan partiendo de lo que los alumnos y alumnas ya conocen, dirigiéndolo hacia los conocimientos nuevos que han de aprender, teniendo en cuenta los intereses propios que manifiesten.

Asimismo, el docente debe propiciar y organizar un ambiente que sea lúdico, agradable, otorgue seguridad y mantenga un clima acogedor y de afecto, con la finalidadde que se establezcan relaciones entre los alumnos y alumnas a través de la actividad infantil en el medio que les rodea, que engloba el juego como motor fundamental para favorecer la socialización.

Por último, cabe destacar la importancia de una buena organización en lo que respecta al espacio 
y al tiempo, en base a todos los aspectos mencionados anteriormente, con el objeto de alcanzar los objetivos propuestos.

Todo ello se ha tenido en cuenta para realizar la secuencia didáctica. A continuación, se presentan diferentes tablas que establecen relación entre los objetivos, contenidos y criterios de evaluación de los que se parte para la puesta en práctica de estapropuesta.
Objetivo general Aplicar la secuencia didáctica para enseñar ciencias de lanaturaleza a través de los cuentos.

Objetivo específico

Objetivo curricular de etapa
Trabajar el concepto de ser vivo a través del pollito y realizaruna analogía con el ser humano.

b) Observar y explorar su entorno familiar, natural y social.

\subsection{ACTIVIDADES DE LA SECUENCIA DIDÁCTICA:}

Cuadro resumen de las actividades que conforman la secuencia didáctica. Fuente: elaboración propia.

\begin{tabular}{|c|c|c|c|}
\hline \multicolumn{4}{|c|}{ FASE 1. Inicial, motivación (exploración y comunicación de objetivos) } \\
\hline \multicolumn{2}{|c|}{ Sesión 1. Ideas previas: ¿qué sabemos? } & \multicolumn{2}{|c|}{$\begin{array}{c}\text { Sesión 2. Cuentacuentos "El pollo Pepe": } \\
\text { ¿qué queremos aprender? }\end{array}$} \\
\hline \multicolumn{4}{|c|}{ FASE 2. Obtención de nuevos conocimientos (confrontación con la realidad) } \\
\hline $\begin{array}{l}\text { Sesión 3. La mamádel } \\
\text { pollito es... }\end{array}$ & $\begin{array}{l}\text { Sesión 4. ¿Dóndeva } \\
\text { cada animal? }\end{array}$ & $\begin{array}{l}\text { Sesión 5. ¿Cómo es el } \\
\text { cuerpo del pollito? } \\
\text { Pero... ¿yel mío? }\end{array}$ & $\begin{array}{l}\text { Sesión } 6 . \text { ¿Comende } \\
\text { todo los pollitos? }\end{array}$ \\
\hline \multicolumn{4}{|c|}{ FASE 3. Estructuración del conocimiento (reflexión y conclusiones) } \\
\hline $\begin{array}{l}\text { Sesión 7. Estampa, } \\
\text { estampa... ¿qué? }\end{array}$ & $\begin{array}{l}\text { Sesión } 8 . \\
\text { ¡Coloreamos quiénvive } \\
\text { en la granja! }\end{array}$ & $\begin{array}{l}\text { Sesión } \\
\text { Rodeando, } \\
\text { rodeando, } \\
\text { tachando, } \\
\quad \text { tachando... }\end{array}$ & $\begin{array}{lr}\text { Sesión 10. } & \text { ¡Hemos } \\
\text { aprendido } & \text { un } \\
\text { montón! } & \end{array}$ \\
\hline \multicolumn{4}{|c|}{ FASE 4. Aplicación (utilización de lo aprendido) } \\
\hline & Sesión 11. ¿Quieres que & te contemos un cuento? & \\
\hline
\end{tabular}

Como se puede ver en la tabla, la secuencia didáctica se encuentra estructurada por sesiones, cada una de ellas correspondiente a una fase de la misma. A continuación, se presentan todas las actividades propuestas. 


\section{- Fase 1. Inicial, motivación (exploración y comunicación de objetivos) -}

\section{Sesión 1. Ideas previas: ¿qué sabemos?}

\section{Objetivos didácticos:}

- Detectar los conocimientos previos que poseen acerca de los pollitos.

- Fomentar la expresión oral.

- Respetar el turno de palabra.

\section{Contenidos:}

- CONCEPTUALES: Huevo.

- PROCEDIMENTALES: Comunicación verbal.

- ACTITUDINALES: Curiosidad, interés, respetar el turno de palabra.

Tipo de agrupamiento: Gran grupo (asamblea).

Materiales: Huevo y pollito de peluche.

Duración: 15-20 minutos aproximadamente.

Desarrollo: Antes de que los alumnos y las alumnas entren al aula el día que se comiencela secuencia, la maestra dejará un huevo* con un pollito dentro en la zona de la asamblea.Los niños y niñas mostrarán curiosidad por el huevo que, de repente, ha aparecido en el aula. Ver anexo I.

La maestra hará la asamblea y, tras finalizarla, con ayuda del encargado o encargada del día, revisaremos qué contiene ese huevo. Con cuidado, el encargado o encargada y la maestra abrirán el huevo para descubrir que ... ¡hay un pollito dentro! Aprovechando esta situación, la maestra realizará una serie de preguntas tales como: ¿quécomen los pollitos?, ¿dónde viven?, ¿cómo es su cuerpo? Se llevará a cabo una lluvia deideas mediada por la maestra en la que los alumnos/as expondrán lo que piensanrespetando el turno de palabra.

*El huevo se realizará con una mezcla de cola y agua a partes iguales y papel de cocina.Se inflará un globo y con trozos pequeños y la mezcla, iremos cubriendo todo el globo con varias capas y, tras dejarlo secar, explotaremos el globo y abriremos por la mitad elhuevo con cuidado para que no se rompa. Dentro meteremos el peluche de "El pollo Pepe" correspondiente al cuento. 
Sesión 2. Cuentacuentos "El pollo Pepe": ¿qué queremos aprender?

\section{Objetivos didácticos:}

- Mantener una escucha activa durante la lectura del cuento.

- Establecer relaciones entre los distintos elementos tratados en el cuento.

\section{Contenidos:}

- CONCEPTUALES: El pollo Pepe, cuento.

- PROCEDIMENTALES: Observación.

- ACTITUDINALES: Escucha activa, interés.

Tipo de agrupamiento: Gran grupo (asamblea).

Materiales: Cuento "El pollo Pepe".

Duración: 10 minutos aproximadamente.

Desarrollo: Una vez que se haya hecho la lluvia de ideas, se presentará al personaje que servirá como hilo conductor para esta secuencia: el pollo Pepe. Es por esto que, tras inspeccionar el huevo, la maestra contará el cuento de "El pollo Pepe" junto al peluche ylos pictogramas de los alimentos que aparecen en él. Ver anexo $I$.

Tras la lectura del cuento les explicaremos a los alumnos y alumnas que el pollitoha venido para enseñarnos cómo vive, lo que come y cómo es su cuerpo.

El cuento se contará a los alumnos y alumnas varias veces durante la secuencia.

\section{- Fase 2. Obtención de nuevos conocimientos (confrontación con la realidad) -}

\section{Sesión 3. La mamá del pollito es...}

\section{Objetivos didácticos:}

- Conocer la relación existente entre pollito-gallina.

- Fomentar el equilibrio corporal. 


\section{Contenidos:}

- CONCEPTUALES: Pollo, gallina (madre e hijo).

- PROCEDIMENTALES: Equilibrio

- ACTITUDINALES: Interés, participación activa.

\section{Tipo de agrupamiento: Gran grupo.}

Materiales: Aros de colores, palitos con símbolo de gallina y de pollito.

Duración: 10-15 minutos aproximadamente.

Desarrollo: Dentro de una sesión de psicomotricidad, una de las actividades consistirá enque a los alumnos y alumnas se les reparte a unos los palos de gallina y a otros los de lospollitos. Ver anexo I. Se les asigna una pareja a cada niño y niña y, en caso de ser impares,dos pollitos a una gallina.

Con los aros colocados por el suelo del patio, debemos explicar a los alumnos y alumnas que, unos son gallinas y otros son los pollitos por lo que, mientras suenen las palmas de las maestras deben ir corriendo o andando por el patio y, cuando dejen de sonar,deben buscar a su mamá gallina y meterse los dos juntos en el mismo aro.

\section{Sesión 4. ¿Dónde va cada animal?}

\section{Objetivos didácticos:}

- Conocer el hábitat del pollito y de distintos animales.

- Fomentar la motricidad fina.

\section{Contenidos:}

- CONCEPTUALES: Animales domésticos, de mar, de aire, de nieve, del bosque, del desierto y de la granja. Águila, cigüeña, búho, oso, delfín, caballito de mar, gato, perro, desierto, serpiente, oso polar, nutria, pollito, gallina.

- PROCEDIMENTALES: Observación de las características, clasificación.

- ACTITUDINALES: Curiosidad, interés, participación. 
Tipo de agrupamiento: Gran grupo (asamblea).

Materiales: Tarjetas didácticas de animales y hábitats.

Duración: 10-15 minutos aproximadamente.

Desarrollo: Estando el alumnado en la zona de la asamblea, se comienza mostrando todaslas tarjetas y nombrando cada uno de los hábitats y animales que en ellas aparecen. Una vez hecho esto, la maestra colocará las tarjetas de los hábitats y los animales en el centrodel espacio. Ver anexo I.

Tras esto y, por turnos, cada uno de los niños y niñas saldrá y escogerá un animalpara colocarlo en su lugar adecuado. Debe decir el nombre del animal y el lugar donde vive. Se hará tantas veces como sea necesario hasta que todos los alumnos y alumnas hayan salido a colocar un animal.

Sesión 5. ¿Cómo es el cuerpo del pollito? Pero... ¿y el mío?

\section{Objetivos didácticos:}

- Conocer el cuerpo de los pollitos y relacionarlo con el propio.

- Fomentar la motricidad fina.

\section{Contenidos:}

- CONCEPTUALES: Partes del cuerpo del pollito: cuerpo, cabeza, alas, patas, ojos, pico, cola, plumas.

Partes del cuerpo del ser humano: cabeza, ojos, nariz, boca, orejas, pelo, cuello,cuerpo, brazos, piernas, pies.

- PROCEDIMENTALES: Observación, experimentación, sujeción adecuada de laspiezas.

- ACTITUDINALES: Interés, participación activa.

Tipo de agrupamiento: Gran grupo (asamblea).

Materiales: Niña y pollito de goma eva.

Duración: 10-15 minutos aproximadamente. 
Desarrollo: Para esta actividad la maestra realiza previamente dos muñecos de goma eva,uno de una niña y otro de un pollito, con todas las partes del cuerpo sueltas, las cuales sesujetan con velcro.

Estando en la zona de la asamblea, la maestra mostrará ambos muñecos y entre todos y todas harán un repaso de las partes del cuerpo tanto del pollito como de la muñeca,estableciendo así una analogía entre ambos. Uno a uno, por turnos, saldrán a colocar unaparte de uno de los muñecos hasta que estén completos. Ver anexo $I$.

\section{Sesión 6. ¿Comen de todo los pollitos?}

\section{Objetivos didácticos:}

- Conocer el alimento de los pollitos.

- Fomentar la motricidad fina.

- Favorecer la clasificación de objetos según consigna.

\section{Contenidos:}

- CONCEPTUALES: Trigo, maíz, cebada.

- PROCEDIMENTALES: Observación, experimentación, clasificación, sujeciónadecuada de la pinza.

- ACTITUDINALES: Participación activa, interés.

Tipo de agrupamiento: Gran grupo (asamblea).

Materiales: Botes con trigo, cebada y maíz, bandeja con gelatina neutra y alimentos yobjetos, bandejas de color rojo y verde, pinzas y pictogramas de los alimentos.

Duración: 20-25 minutos aproximadamente.

Desarrollo: Situando a los alumnos y alumnas en la zona de la asamblea, les enseñaremosel maíz, la cebada y el trigo junto a unas tarjetas con el pictograma de cada uno de los alimentos.

Posteriormente y cuando identifiquen cada uno de ellos, se les mostrará una bandeja rellena de 
gelatina neutra dentro de la cual podremos encontrar tanto la comida de los pollitos (trigo, maíz, cebada), como macarrones, unas bolitas procedentes de los árboles y unos huevos de pascua de juguete de tamaño pequeño.

La actividad consiste en que, con ayuda de unas pinzas, cada uno de los niños y niñas sacará algo y deberá colocarlo en su bandeja correspondiente: verde para las cosas que sí comen los pollitos y roja para las cosas que no, diciendo además lo que ha cogidoy mostrándolo a los demás. Ver anexo I.

\section{- Fase 3. Estructuración del conocimiento (reflexión y conclusiones) -}

\section{Objetivos didácticos:}

- Reforzar el esquema corporal del pollito.

- Fomentar la motricidad fina.

- Favorecer el desarrollo de la creatividad a través del estampado.

\section{Contenidos:}

- CONCEPTUALES: Cuerpo, cabeza, alas, patas, ojos, pico, cola, plumas.

- PROCEDIMENTALES: Experimentación, estampación, sujeción adecuada delos sellos.

- ACTITUDINALES: Participación activa.

Tipo de agrupamiento: Individual (grupos de trabajo).

Materiales: Sellos de distintos tamaños: círculo grande, círculo pequeño, triángulo ypatas, pintura naranja y amarilla, rotulador negro, folio o cartulina blanca.

Duración: 20-25 minutos aproximadamente.

Desarrollo: Todos los alumnos y alumnas se sentarán en sus mesas de trabajo y, con ayudade la maestra tutora y la maestra de apoyo, irán estampando los sellos en orden: cuerpo- cabeza-patas-pico. Ver anexo I.

Una vez que esté seco, con un rotulador negro los alumnos y alumnas pintarán losojos a los pollitos. 


\section{Sesión 8. ¡Coloreamos quién vive en la granja!}

\section{Objetivos didácticos:}

- Reforzar el hábitat del pollito.

- Fomentar la motricidad fina.

\section{Contenidos:}

- CONCEPTUALES: Granja, pollito, gallina.

- PROCEDIMENTALES: Sujeción adecuada del rotulador, observación.

- ACTITUDINALES: Interés, participación activa.

\section{Tipo de agrupamiento: Individual (grupos de trabajo).}

\section{Materiales: Ficha.}

Duración: 15-20 minutos aproximadamente.

Desarrollo: Cuando todo el alumnado se encuentre sentado en su mesa de trabajo, se lesrepartirá la ficha, previamente explicada en la asamblea. Dicha ficha consiste en colorear de entre todos los animales que aparecen al lado de la granja, cuáles de ellos viven enella. Opcionalmente se puede tachar aquel animal que no vive en la granja. Ver anexo I.

\section{Sesión 9. Rodeando, rodeando, tachando, tachando...}

\section{Objetivos didácticos:}

- Reforzar el conocimiento de los alimentos que come el pollito.

- Fomentar la motricidad fina.

\section{Contenidos:}

- CONCEPTUALES: Trigo, cebada, maíz.

- PROCEDIMENTALES: Sujeción adecuada del rotulador, observación.

- ACTITUDINALES: Participación activa e interés. 
Tipo de agrupamiento: Individual (grupos de trabajo).

Materiales: Ficha.

Duración: 15-20 minutos aproximadamente.

Desarrollo: En el momento en el que todos los niños y niñas estén sentados, se repartirá la ficha que previamente ha sido explicada en la asamblea. Esta ficha se compone de alimentos que comen los pollitos y otras cosas que no, por lo que los alumno y alumnas deben rodear aquello que sí comen y tachar lo que no. Ver anexo $I$.

\section{Sesión 10. ¡Hemos aprendido un montón!}

\section{Objetivos didácticos:}

- Detectar si se han aprendido los contenidos.

- Favorecer el desarrollo de la expresión oral.

- Respetar el turno de palabra.

\section{Contenidos:}

- CONCEPTUALES: Huevo, animales domésticos, de mar, de aire, de nieve, del bosque, del desierto y de la granja, águila, cigüeña, búho, oso, delfín, caballito demar, gato, perro, desierto, serpiente, oso polar, nutria, pollito, gallina, trigo, cebada, maíz, cuerpo del pollito: cuerpo, cabeza, alas, patas, ojos, pico, cola, plumas, cuerpo del ser humano: cabeza, ojos, nariz, boca, orejas, pelo, cuello, cuerpo, brazos, piernas, pies.

- PROCEDIMENTALES: Comunicación oral.

- ACTITUDINALES: Participación, interés, respetar el turno de palabra.

Tipo de agrupamiento: Gran grupo (asamblea).

Materiales: No precisa.

Duración: 10-15 minutos aproximadamente. 
Desarrollo: Estando situados en la zona de la asamblea, la maestra iniciará un turno depalabra en el que los alumnos y alumnas deben responder a lo que la maestra pregunta.

Estas cuestiones son para comprobar que los conocimientos han sido adquiridos por todo el alumnado. Se formularán aquellas preguntas realizadas en la sesión de ideas previas, con el fin de hacer un recorrido por todos los contenidos tratados, desde el primerdía con la aparición del huevo, el cuerpo del pollito, el cuerpo humano, la comida del pollito hasta el hábitat del pollito.

\section{- Fase 4. Aplicación (utilización de lo aprendido) -}

\section{Sesión 11. ¿Quieres que te contemos un cuento?}

\section{Objetivos didácticos:}

- Favorecer el desarrollo de la expresión oral.

- Establecer relaciones entre los distintos elementos tratados en el cuento.

\section{Contenidos:}

- CONCEPTUALES: Contenidos que aparecen en el cuento de El pollo Pepe: pollo,maíz, cebada, trigo, patas, pico, barriga, gallina.

- PROCEDIMENTALES: Comunicación oral.

- ACTITUDINALES: Participación activa, interés.

\section{Tipo de agrupamiento: Gran grupo (asamblea).}

\section{Materiales: Cuento "El pollo Pepe".}

\section{Duración: 10 minutos aproximadamente.}

Desarrollo: Estando todos los niños y niñas en la zona de la asamblea, la maestra comenzará a contar el cuento y, en cada palabra importante y significativa del cuento, sequedará callada para que, entre todos los alumnos y alumnas, cuenten el cuento junto a lamaestra.

Inicialmente, la idea propuesta era que los alumnos y alumnas del aula de 3 años cuenten al resto de clases de Educación Infantil el cuento. Sin embargo, debido a la situación en la que nos encontramos ocasionada por el virus de Covid-19, esto no ha sidoposible por los grupos burbuja que se han de mantener. 


\section{Evaluación:}

Conforme a lo establecido en el artículo 7 del Real Decreto 1630/2006, de 29 de diciembre, por el que se establecen las enseñanzas mínimas del segundo ciclo de Educación Infantil, la evaluación en esta etapa debe ser "global, continua y formativa. Laobservación directa y sistemática constituirá la técnica principal del proceso de evaluación” (p. 475).

Asimismo, se recoge en el artículo 6 del Decreto 254/2008, de 1 de agosto por elque se establece el currículo del segundo ciclo de la Educación Infantil en la ComunidadAutónoma de la Región de Murcia, que la evaluación en Educación Infantil "debe servirpara identificar los aprendizajes adquiridos y el ritmo y características de la evolución decada niño o niña” (p. 24962).

De tal forma que se establecen tres momentos de evaluación durante la secuenciade aprendizaje:

En primer lugar, se lleva a cabo una evaluación inicial de los conocimientos que poseen los alumnos y alumnas antes de comenzar la puesta en práctica. Dicha evaluaciónse lleva a cabo mediante la Sesión 1, correspondiente a la Fase 1, en la que, a través de preguntas en la asamblea, podremos saber desde qué punto parte el alumnado.

En segundo lugar, durante el proceso de enseñanza-aprendizaje, se realiza una evaluación procesual a través de la observación directa y sistemática, teniendo como referencia las escalas de estimación: una serie de rúbricas con indicadores de evaluaciónen relación a las actividades. Un ejemplo de rúbrica sería el siguiente:

Indicadores de evaluación de la Sesión 2.

\begin{tabular}{|c|c|c|c|}
\hline Indicador de evaluación & Conseguido & En proceso & No conseguido \\
\hline
\end{tabular}

el cuento.

Reconoce los elementos tratados en

Fuente: elaboración propia.

A continuación, se enumeran los indicadores de evaluación de todas las actividades:

- Participa en la conversación.

- Se interesa por expresarse oralmente.

- Respeta el turno de palabra.

- Se interesa por el relato que se está contando.

- Reconoce los elementos tratados en el cuento.

- Reconoce la relación entre pollito-gallina. 
- Controla su equilibrio corporal.

- Distingue las diferencias entre los hábitats y los diferentes animales.

- Ejercita la habilidad motriz fina.

- Es capaz de reconocer las partes del cuerpo de los pollitos y las suyas propias.

- Muestra interés por conocer los distintos alimentos de los pollitos.

- Clasifica correctamente según consigna.

- Conoce el esquema corporal del pollito y el propio.

- Se interesa por la estampación.

- Comprende cuál el hábitat del pollo.

- Sabe cuál es el alimento que comen los pollitos.

- Da muestras de conocer los contenidos con soltura.

- Comprende las relaciones que se establecen entre los elementos del cuento.

En tercer lugar, a través de la Sesión 11, correspondiente a la Fase 4, comprobaremos, de manera global y tras ver los resultados obtenidos en dicha actividad y en la evaluación procesual si los alumnos y alumnas han conseguido los objetivos que se pretendían con la secuencia de enseñanza. Todo ello se recoge en una rúbrica y un gráfico que se mostrará más adelante, con los resultados finales de la secuencia en funciónde cuántos alumnos y alumnas han conseguido los objetivos de las sesiones.

\section{Indicadores de evaluación final.}

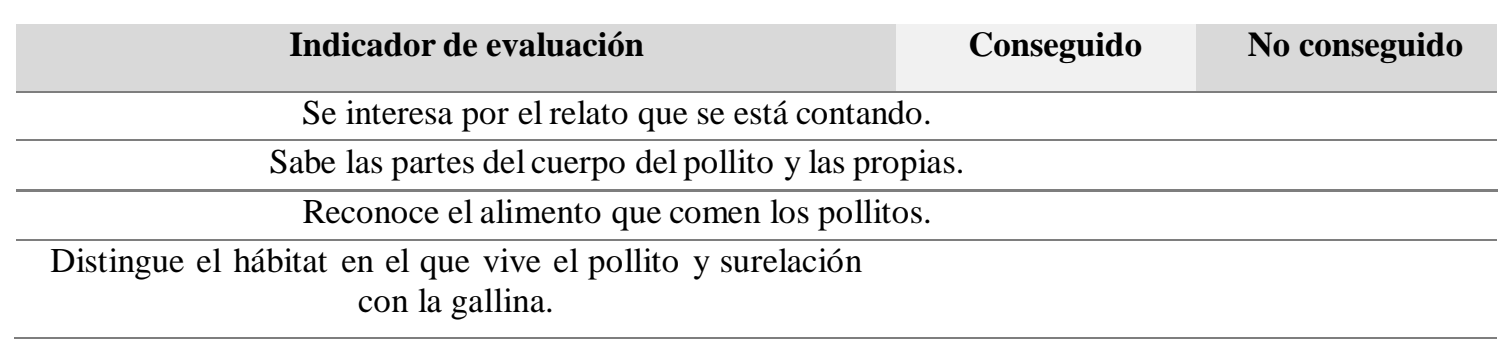

Tal y como especifica el artículo 13 de la Orden de 22 de septiembre de 2008, dela Consejería de Educación, Ciencia e Investigación, por la que se regulan, para la Comunidad Autónoma de la Región de Murcia, la implantación, el desarrollo y la evaluación en el segundo ciclo de la Educación Infantil, "los maestros evaluarán los procesos de enseñanza y su práctica docente con la finalidad de mejorarlos y adecuarlos a las características específicas y a las necesidades educativas de los alumnos” (p. 31237).

Por tanto, para llevar a cabo una autoevaluación, tendremos en cuenta los siguientes aspectos: 
- La planificación general, es decir, si se han obtenido los resultados propuestos enlo que respecta a lo se planifica previamente que se va a realizar y lo que finalmente se lleva a cabo.

- La organización del tiempo, que se encuentra ligada a las características del alumnado y los tiempos que necesite.

- La organización del espacio teniendo en cuenta las actividades propuestas.

- Elaboración de materiales: si tienen el tamaño adecuado, si son llamativos o si sonútiles.

\subsection{REFLEXIÓN SOBRE LA PUESTA EN PRÁCTICA:}

A continuación, se lleva a cabo una reflexión sobre esta puesta en práctica que fuellevada a cabo por mí personalmente en mis últimas prácticas de la universidad.

De acuerdo a lo mencionado por Pérez Molina, et al. (2013) en el apartado del marco teórico, he podido comprobar cómo, efectivamente, se produce una gran interacción entre los niños y niñas y la persona que cuenta el cuento, en este caso yo. A pesar de ser un cuento simple, el alumnado mostró gran curiosidad por ver qué seguía y qué se contaba sobre el pollo Pepe en dicho cuento. Además, hacían comentarios que ibanmás allá de lo que podían ver, lo cual da a entender que, efectivamente, los niños y niñassaben más de lo que podemos llegar a pensar.

Fue grato para mí, comprobar que, con tan poco se puede lograr mucho, pues el resultado final de la secuencia no ha hecho más que sorprenderme favorablemente. Tras la realización de la secuencia didáctica y una reflexión individual posterior, puedo afirmar que esta puesta en práctica ha sido satisfactoria en lo que respecta a la consecución de miobjetivo general del trabajo, ya que, finalmente, tras los resultados recogidos en el gráficoque se presenta a continuación, podemos apreciar que, en la última fase de la secuencia, todo el alumnado fue capaz de realizar la actividad final sin ningún problema, evidenciando los conocimientos adquiridos que se proponían con las sesiones. Además, en ninguna de las actividades hubo un alumno o alumna que no fuera capaz de realizarla,por lo que considero que es un punto a favor de esta propuesta. 
Resultados de la secuencia de aprendizaje.

\section{Resultados secuencia de aprendizaje}

25

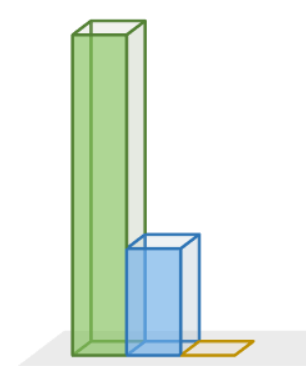

Fase 1
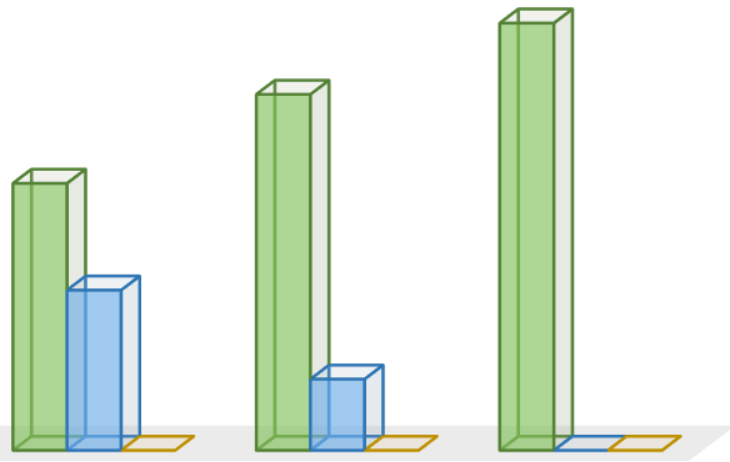

Fase 2

Fase 3

Fase 4

$\square$ Conseguido $\square$ En proceso $\square$ No conseguido

Fuente: elaboración propia.

\section{CONCLUSIONES, CONSECUENCIAS E IMPLICACIONES EDUCATIVAS}

En conclusión, en base al objetivo marcado, podemos afirmar que se ha logrado un acercamiento a la naturaleza en esta aula de 3 años, al conseguir que los alumnos y alumnas adquieran los conocimientos referidos al objetivo de la secuencia, el cual es trabajar el concepto de ser vivo a través del pollito y realizar una analogía con el ser humano a través del cuento. Los alumnos y alumnas han terminado la secuencia de actividades sabiendo discernir entre los distintos aspectos que nos diferencian de los pollitos, en relación al esquema corporal, la alimentación y el hábitat de cada uno.

Esto quedó reflejado en la Sesión 10, en la que se realizó una asamblea para que,entre todos, comentaran qué habían aprendido a través de una serie de preguntas por mi parte, que fueron las mismas que se utilizaron en la Sesión 1, con el fin de obtener diferentes respuestas habiendo hecho las mismas preguntas.

En lo que respecta a la utilización de otros materiales y otras actividades para no llevar a cabo el método tradicional, he de decir que, aunque requiere de mayor esfuerzo por parte del docente, es mucho más enriquecedor para ellos y también para el propio docente, porque se es consciente de que existen otras maneras de enseñar y de que los niños y niñas aprendan. Con la mayor parte de mis actividades los alumnos y alumnas han demostrado una actitud adecuada para el aprendizaje a la par que lúdica, pues no es lo mismo pintar un pollito con rotulador que realizarlo con sellos hechos a mano que sondistintos a los que se comercializan.

En cuanto a mejoras que haría si tuviera que realizar de nuevo esta secuencia didáctica, me centraría en realizar unos materiales de mayor tamaño ya que, a pesar de que en este caso no ha supuesto 
un problema para los niños y niñas porque la mayor partede las propuestas se han realizado en la asamblea, no todas los alumnos y alumnas son iguales, por lo que considero que este aspecto es importante tenerlo en cuenta.

Además, como actividad añadida que puede ser muy motivadora e interesante parael alumnado, sería proponer al centro una actividad complementaria en la que se fuera a una granja escuela, donde el alumnado pudiera ver en primera persona pollitos de verdady, ser conscientes también, de su tamaño real. Esto no ha podido ser posible en esta secuencia debido a la situación de pandemia mundial que atravesamos ocasionada por elvirus de Covid-19.

Por último, me gustaría mandar un mensaje a todos los futuros docentes y a los que ya lo son: a los niños y las niñas les gustan las ciencias, les gusta aprender, hagámoslofácil y divertido para que, en el futuro, dejemos huella en ellos y crezcan sabiendo valorartodo lo que tienen a su alrededor, por pequeño que sea.

'A los niños, antes de enseñarles a leer,

hay que ayudarles a aprenderlo que es el amor y la verdad'

\section{(Mahatma Gandhi)}




\section{REFERENCIAS}

Cabrera, G. (2001). Y va de cuentos. Letras Libres, 33. Recuperado de https://www.letraslibres.com/mexico/y-va-cuentos

Chevallard, 1991. Citado por Gutiérrez Abbad, A. (s.f.). Sesión 1, Las Ciencias de la Naturaleza en el currículo de Educación Infantil. Material no publicado.

Decreto 254/2008, de 1 de agosto, por el que se establece el currículo del Segundo Ciclode la Educación Infantil en la Comunidad Autónoma de la Región de Murcia. Boletín Oficial de la Región de Murcia, 182, 06 de agosto, pp. 24960-24973.

Denchfield, N. (1997). El pollo Pepe. Editorial SM.

Espinet Blanch, M. (1995). El papel de los cuentos como medio de aprendizaje de las ciencias en la educación infantil. Aula de innovación educativa, 44, 01-05. Recuperado de https://ddd.uab.cat/record/183067

Fernández Serón, C. G. (2010). El cuento como recurso didáctico. Innovación y experiências educativas, 26 , 01-09.

Recuperado de

https://archivos.csif.es/archivos/andalucia/ensenanza/revistas/csicsif/revista/pdf/

Nume ro_26/CRISTINA_GEMA_FERNANDEZ_SERON_01.pdf

Imbert, E. A. (1979). Teoría y técnica del cuento. Buenos Aires, Argentina: Ediciones Marymar. Recuperado de https://www.academia.edu/download/59884002/Anderson_Imbert E__Teoria_y_Tecnica_del_Cuento20190627-39913-1bclcgg.pdf

López Cassá, E. (2005). La educación emocional en la educación infantil. Revista Interuniversitaria de Formación para el Profesorado, 19, 153-167. Recuperado de https://www.redalyc.org/pdf/274/27411927009.pdf

Martínez Urbano, N. (2011). El cuento como instrumento educativo. Innovación y experiências educativas, 39, 01-08. Recuperado de https://archivos.csif.es/archivos/andalucia/ensenanza/revistas/csicsif/revista/pdf/_ Nume ro_39/NATALIA_MARTINEZ_URBANO_01.pdf

Muñoz Muñoz, A., y Díaz Perea, M. R. (2009). Metodología por proyectos en el área deconocimiento del medio. Docencia e Investigación, 19, 101-126. Recuperado de https://www.researchgate.net/profile/Alberto_Munoz7/publication/45588718_Metodologia_por_proye ctos_en_el_area_de_conocimiento_del_medio/links/54b684cb0cf2e68eb27e9982/Metodologia-porproyectos-en-el-area-de-conocimiento-del-medio.pdf

Orden de 22 de septiembre de 2008, de la Consejería de Educación, Ciencia e Investigación, por la que se regulan, para la Comunidad Autónoma de la Región de Murcia, la implantación, el desarrollo y la evaluación en el segundo ciclo de la Educación Infantil. Boletín Oficial de la Región de Murcia, 238, 11 de octubre, 31233-31239.

Pérez Molina, D., Pérez Molina, A. I., y Sánchez Serra, R. (2013). El cuento como recursoeducativo. 3c Empresa: investigación y pensamiento crítico, 13, 02-29. Recuperado de https://dialnet.unirioja.es/servlet/articulo?codigo $=4817922$ 
Real Academia Española. (s.f.). Cuento. En Diccionario de la lengua española. Recuperado en 11 de mayo de 2021, de https://dle.rae.es/cuento

Real Decreto 1630/2006, de 29 de diciembre, por el que se establecen las enseñanzas mínimas del segundo ciclo de Educación infantil. Boletín Oficial de la Región deMurcia, 4, 04 de enero, 474-482.

Toledo Morales, P. (2005). El valor educativo del cuento: didáctica y evolución histórica. Recuperado de https://www.researchgate.net/profile/Purificacion-Toledo

Morales/publication/324774827_El_valor_educativo_del_cuento_didactica_y_evolución_historica/link s/5ae1a736aca272fdaf8e226c/El-valor-educativo-del-cuento-didactica-y-evolucion-historica.pdf

Veglia, S. M. (2007). Ciencias naturales y aprendizaje significativo: claves para la reflexión didáctica y la planificación. Buenos Aires, Argentina: Ediciones Noveduc 